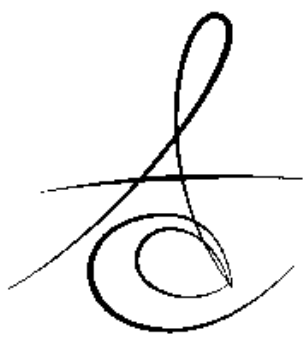

\section{RENKLENMİŞ KÖK KANAL TEDAVİLİ SOL ÜST ÇENE LATERAL VE KANİN DİŞLERİN TEDAVİSi: OLGU SUNUMU\#}

TREATMENT OF DISCOLORATION OF MAXILLARY LEFT LATERAL INCISOR AND CANINE: CASE REPORT ${ }^{\#}$

\author{
Dr. Dt. Buğra GÜLER ${ }^{*}$
}

Arş. Gör. Dr. Dt. Taha ÖZYÜREK *

\author{
Yrd. Doç. Dr. İsmail UZUN*
}

Makale Kodu/Article code: 1684

Makale Gönderilme tarihi; 107.05.2014

Kabul Tarihi: 11.11.2014

\section{ÖZET}

Dişlerde oluşan renklenmeler lokalizasyonuna ve etyolojisine bağlı olarak iç ve dış kaynaklı veya her ikisinin kombinasyonu olarak sınıflandırılabilir. Renklenmiş dişlerin tedavisinde, tam seramik kronlar, metal destekli porselen kronlar ve kompozit veya porselen lamineler-invaziv yöntemlere başvurulmadan önce, doğru teşhis konularak beyazlatma tedavileri uygulanabilir. Beyazlatma tedavilerinde oldukça kolay uygulanabilen materyal ve yöntemler geliştirilmesi yanında, daha konservatif tedavi yöntemi olması nedeniyle kimyasal ajanlarla beyazlatma tercih edilmektedir. Beyazlatma mekanizması açık olmamakla beraber temeli oksidasyona dayanır. Diş hekimliğinde beyazlatma ajanları genellikle hidrojen peroksitin çeşitli formlarından oluşmaktadır. Bu amaçla hidrojen peroksit, sodium perborat, karbamid peroksit gibi ajanlar kullanılır. Bu çalışmanın amacı, renklenmiş devital sol üst çene lateral ve kanin dişlerde walking bleaching tekniği ile \%37'lik karbamit peroksit beyazlatma ajanının etkinliğinin değerlendirilmesi ve 2 yıllık takibinin sunulmasıdır.

Anahtar kelimeler: Walking bleaching, devital diş, karbamit peroksit

\begin{abstract}
Discoloration of teeth can be classified according to localization and etiology as internal, external or combined. Before applying conventional invasive techniques such as all ceramic crown, composite, porcelain laminates and porcelain-metal crowns; bleaching can be attempted when indicated. Bleaching with chemical agents is preferred with advantages such as being conservative and development of novel materials and methods. Mechanism of bleaching is not clear but it depends on oxidation reaction. Bleaching agents used in dentistry consist various forms of hydrogen peroxide. Agents such as hydrogen peroxide, sodium perborate and carbamide peroxide are applied. Aim of this case report is to present the efficacy of $37 \%$ carbamide peroxide bleaching agent with the treatment of discolored devital teeth and follow-up.
\end{abstract}

Key words: walking bleaching, devital tooth, carbamide peroxide

\section{GİRİş}

Çeşitli nedenlerle dişlerde oluşan renklenmeler özellikle anterior bölgede estetik problemlere yol açmaktadır. Birçok etken dişlerde renklenmeye neden olabilmektedir. Lokalizasyonuna ve etyolojisine bağlı olarak renklenmeler, iç ve dış kaynaklı olarak veya her ikisinin kombinasyonu şeklinde gözlenebilir. ${ }^{1,2}$ Dış kaynaklı renklenmeler çay, kahve, meyve suyu, sigara çeşitli ağız gargaraları gibi etkenlerin diş yüzeyinde birikmesi sonucunda oluşur. ${ }^{1,3}$ İç kaynaklı renklenmelerin nedenleri ise sistemik ve lokal faktörler olmak üzere iki bölümde incelenmektedir. İlaçlar (tetrasiklin gibi), distrofik kalsifikasyonlar, florozis, hiperbilirubinemi, amelogenezis imperfekta, dentinogenezis imperfekta sistemik faktörler arasında sayılabilir. ${ }^{4}$ Pulpa nekrozu, intrapulpal hemoraji, endodontik tedavi sonrası kalan artık pulpa dokuları, endodontik

\footnotetext{
${ }^{a}$ Ondokuz Mayıs Üniversitesi Diş Hekimliği Fakültesi Endodonti Anabilim Dalı

\# Türk Endodonti Derneği 5. Bilimsel Sempozyumu'nda poster olarak sunulmuştur

01-04 Haziran 2013/ İzmir, Türkiye
} 
materyaller, koronal restorasyon materyalleri, kök rezorpsiyonu ve yaşlanma gibi nedenler de iç kaynaklı renklenmelerin lokal faktörleri arasında sayılabilir. ${ }^{4}$

Renklenmiş dişlerin tedavisinde uygulanan beyazlatma tedavileri, kompozit ve porselen lamineler, metal destekli porselen ve tam seramik restorasyonlarla yapılan geleneksel tedavilere.$^{5}$ göre daha konservatif bir yaklaşımdır. Tam seramik restorasyonların planlandığı vakalarda ilgili dişe restorasyondan önce yapılan ağartma işlemi de estetik sonucun başarııını arttıracaktır. ${ }^{4}$

Devital dişlerin tedavisinde termokatalitik yöntem, walking bleaching tekniği, ev tipi ve ofis tipi ağartma veya kombine teknikler kullanılabilir. ${ }^{5,6}$ Termokatalitik teknikte \%30-35'lik hidrojen peroksit pulpa odasına yerleştirildikten sonra ISı ile aktive edilir.7 Ancak bu yöntemin beyazlatma işlemi sonrasında meydana gelen eksternal servikal kök rezorpsiyonuna neden olduğu düşünülmektedir. ${ }^{8,9}$

Günümüzde devital dişlerin beyazlatılmasında en çok kullanılan yöntem walking bleaching tekniğidir. Bu teknikte genellikle sodyum perborat patı, hidrojen peroksit veya su ile karışıırıarak pulpa odasına yerleştirilir ve diş geçici olarak restore edilir. Bu işlem 3 ila 7 gün aralıklarında istenilen sonuç alınıncaya tekrarlanır. ${ }^{7,9-11}$ Tekniğin sıklıkla kullanılmasının nedeni uygulamanın basit, hızlı ve etkin olmasının yanı sıra ${ }^{12,13}$ klinik olarak güvenilir olmasıdır. ${ }^{11,14}$

$\mathrm{Bu}$ olguda endodontik tedavi sonucu renklenmiş sol üst çene lateral ve kanin dişlerinin walking bleaching tekniği ile beyazlatılması ve 2 yıllık takibinin sunulması amaçlanmaktadır.

\section{OLGU SUNUMU}

44 yaşındaki bayan hasta sol üst çene lateral ve kanin dişlerindeki renklenme şikayeti ile Ondokuz Mayıs Üniversitesi Diş Hekimliği Fakültesi Endodonti Anabilim Dalı'na başvurdu. Yapılan klinik ve radyolojik muayeneler sonucunda dişlerin kanal tedavili olduğu ve kronların bukkal ve palatinal yüzeylerinde yaygın renklenmelerin bulunduğu gözlendi.. Renklenmenin giderilebilmesi için "walking bleaching" tekniği ile beyazlatma uygulanmasına karar verildi. İlgili dişlerin beyazlatma öncesindeki renklenme derecelerini tedavi sonrası ile karşısaştırmak amacıyla işlem öncesinde dişlerden uygun açılarla fotoğraflar (Canon PowerShot A2200, Japan) alındı. (Resim 1,2) Dişlerin rubber dam ile izole edilmesinin ardından, uygun giriş kaviteleri hazırlandı ve kök kanal dolguları mine sement bileşiminin $2 \mathrm{~mm}$ altında kalacak şekilde çelik rond frezle (Dentsply Maillefer, Ballaigues, Switzerland) uzaklaştırıldı. Bu işlem sırasında meydana gelen smear tabakasının kaldırıması için kavite içerisindeki dentin $\% 5.25^{\prime}$ lik sodyum hipoklorit solüsyonunun ardından $\% 17^{\prime}$ lik EDTA solüsyonu ile yıkandı ve kurulandı. Koroner bölgede oluşabilecek sızıntıları önlemek için 2 $\mathrm{mm}$ kalınlığında cam iyonomer siman (Fuji IX, GC Corporation, Tokyo, Japan) kanal ağızlarına yerleştirildi.

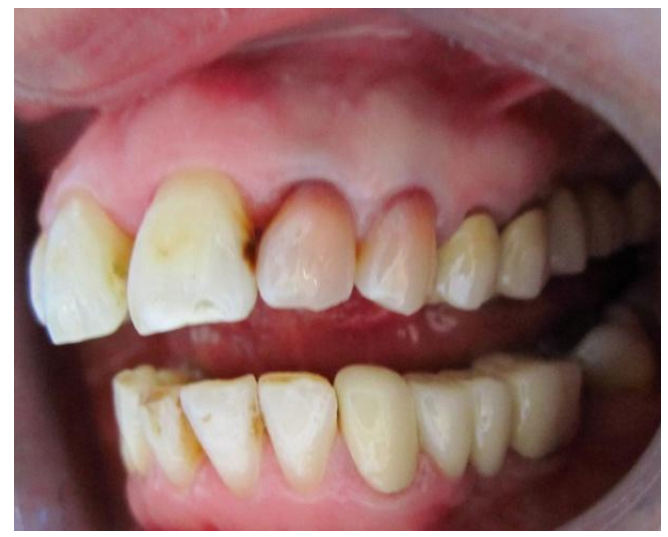

Resim 1. Renklenmiş devital sol üst çene lateral ve kanin dişlerin tedavi öncesi görüntüsü.

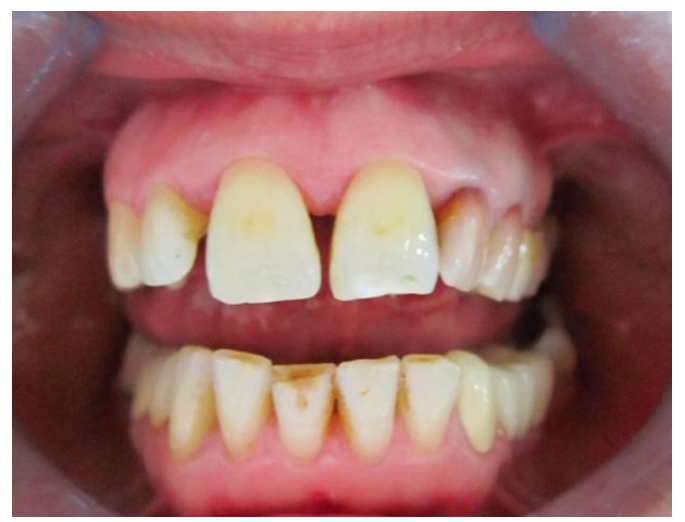

Resim 2. Renklenmiş devital sol üst çene lateral ve kanin dişlerin tedavi öncesi görüntüsü.

Devital beyazlatma ajanı pulpa odalarına uygulanmadan önce kavite içerisindeki mine dokusuna \%37'lik ortofosforik asit uygulandı. Devital beyazlatma ajanı olarak \%37'lik karbamid peroksit içeren hazır preperat (Whiteness Super Endo, FGM Produtos Odontologicos, Joinvile, Brazil) pulpa odalarına uygu- 
landıktan sonra üzerlerine steril pamuk peletler yerleştirildi ve kaviteler geçici olarak ışıkla sertleşen kompozit rezin (Gradia GC, Tokyo, Japan) ile restore edildi. Dört gün sonraki ikinci seansta dişlerdeki renklenmelerin kısmi olarak azaldığı, ancak tam olarak ortadan kalkmadığı görüldüğü için beyazlatma ajanı yenilendi ve hastaya 4 gün sonrasına randevu verildi. Üçüncü seans sonunda dişlerinin istenilen düzeyde beyazladığı gözlendi.(Resim 3)

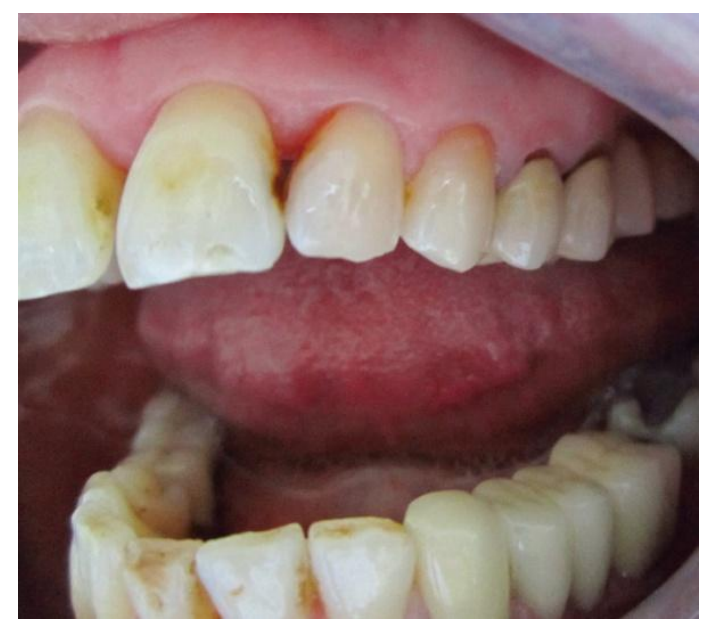

Resim 3. Sol üst çene lateral ve kanin dişlerin tedavi sonrası görüntüsü.

Kullanılan beyazlatma ajanının distile su ile yıkanarak pulpa odasından uzaklaştırımasının ardından, karbamit peroksitin etkinliğini nötralize etmek için kavitelere kalsiyum hidroksit $\left(\mathrm{Ca}(\mathrm{OH})_{2}\right)$ patı (Calcicure, Voco, Germany) uygulandı ve üzerlerine steril pamuk peletler yerleştirilerek kaviteler geçici olarak ışıkla sertleşen kompozit rezinle restore edildi. Bir hafta sonra yapılan dördüncü seansta kavite içerisinde kalan $\mathrm{Ca}(\mathrm{OH})_{2}{ }^{\prime}$ in uzaklaştııııması sonrasında dişler daimi olarak ışıkla sertleşen kompozit rezin materyali (Gradia GC, Tokyo, Japan) ile restore edildi.

İki yıl sonra yapılan kontrolde hastanın herhangi bir rahatsızıı̆ının olmadığı, dişlerin renklerinin stabil kaldığı gözlendi.(Resim 4,5) Ayrıca yapılan radyolojik muayenede herhangi bir patolojiye rastlanmadı.

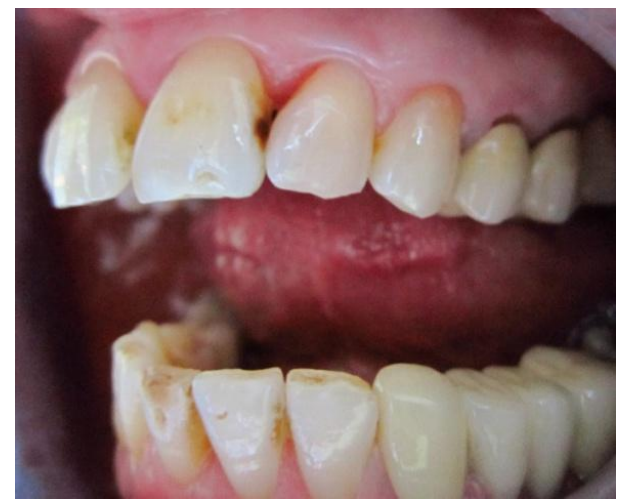

Resim 4. Sol üst çene lateral ve kanin dişlerin tedaviden 1 ay sonraki görüntüsü.

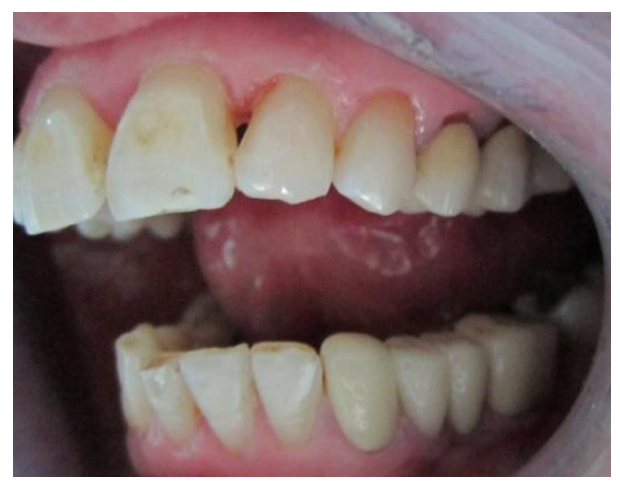

Resim 5. Sol üst çene lateral ve kanin dişlerin tedaviden 2 yıl sonraki görünümü.

\section{TARTIŞMA}

Beyazlatmanın mekanizması açık olmamakla beraber temeli oksidasyona dayanır. Diş hekimliğinde kullanılan beyazlatma ajanları genellikle hidrojen peroksit, sodyum perborat, karbamid peroksit gibi hidrojen peroksitin çeşitli formlarından oluşmaktadir. $^{7,10,15}$ Karbamid peroksit içeren preperatların da hidrojen peroksit ve sodyum perborat kadar etkili oksidasyon yeteneğine sahip olduğu bilinmektedir.. ${ }^{10,16}$ Karbamid peroksit dokularla temas ettiği zaman üre, amonyak, karbondioksit, hidrojen peroksit ve preperatın konsantrasyonuna bağlı olarak farklı düzeylerde oksijen açığa çıkar..$^{9,10,15,16}$

Beyazlatma işlemi için kullanılan çeşitli tekniklerden biri olan hidrojen peroksitle beraber kullanılan termokatalitik yöntemde açığa çıkan kostik maddeler dişin servikalinde $\mathrm{pH}^{\prime}$ nin düşmesine ve bunun sonucunda da servikal kök rezorbsiyonuna neden olurlar. ${ }^{8,9,17} \mathrm{Bu}$ nedenle günümüzde devital dişlerin 
bayazlatılması sırasında termokatalitik yöntem tercih edilmemekte walking bleaching tekniği ise sıklıkla kullanılmaktadır. ${ }^{18}$

Beyazlatma tedavisi sırasında meydana gelen $\mathrm{pH}$ değişimlerinin neden olduğu servikal kök rezorpsiyonunun önlenebilmesi amacıyla, sodyum perborat veya karbamid peroksit kullanılabilir. ${ }^{5}$ Beyazlatma ajanlarının kök dışına difüzyon oranlarını inceleyen bir çalışmada $\% 35^{\prime}$ lik hidrojen peroksitin en fazla, sodyum perboratın orta düzeyde ve karbamid peroksitin ise en az düzeyde kök dışına difuze olduğu gösterilmiştir. ${ }^{19}$ Karbamid peroksitin düşük difüzyon düzeyi ve beyazlatmadaki başarısı ${ }^{20}$ dikkate alındığında diğer preperatlara oranla kullanımının daha güvenli olduğu görülmektedir. Bu sebeplevakamızda walking bleaching tekniğiyle \%37'lik karbamid peroksit kullanılmayı tercih ettik.

Beyazlatma ajanlarının apikal bölgeye ulaşmasını engellemede kök kanal dolgusunun yeterli olmadığı bilindiğinden ${ }^{21,22}$ Hansen-Bayless ve Davis ${ }^{23}$ kaide maddelerinin kullanımını önermişlerdir. Bu amaçla cam iyonomer simanlar, IRM (intermediate restorative material), hidrofilik materyaller, kompozit resinler, çinko oksit ojenol ve çinko fosfat simanlar kullanılmaktadır. ${ }^{4}$ Cam iyonomer simanlar ile yapılan bir çalışmada $2 \mathrm{~mm}$ kalınlığındaki cam iyonomer simanın \%30'luk hidrojen peroksit solüsyonunun apikale difüzyonunu önlediği gösterilmiştir. ${ }^{24} \mathrm{Bu}$ nedenle vakamıda cam iyonomer siman kaide materyali olarak kullanılmıştır.

Beyazlatma işleminden sonra dişlerin mikroorgzanizmalarla tekrar enfekte olmasını, boyayıcı maddelerin dişe tekrar sızmasını önlemek ve dişin dayanıkılığını arttırmak ${ }^{25,26}$ amacıyla dişler mine ve dentine oldukça yüksek adezyon özelliğine sahip kompozit rezinler ile restore edilmelidir. Beyazlatma işleminden sonra kavitede kalan peroksit yada oksijenin kompozit rezinlerin polimerizasyonunu inhibe ettiği yapılan çalışmalarda gösterilmiştir. ${ }^{27,} 28$ Ayrıca beyazlatma işlemi sonucunda minenin yapısında oluşan değişiklikler kompozit rezinlerin adezyonunu olumsuz yönde etkileyebilir. ${ }^{28} 29 \mathrm{Bu}$ sebeple beyazlatma işleminden sonra pulpa odasına yerleştirilen kalsiyum hidroksit patı, asidik pH'nın tamponlanması ve olası servikal kök rezorpsiyonun önüne geçilmesi için bir hafta süre ile uygulanmalıdır. ${ }^{25,} 30$ Yapılan çalışmalarda pulpa odasına uygulanan kalsiyum hidroksitin daimi restorasyon için kullanılan kompozit rezinlerin bağlantısını etkilemediği gösterilmiştir. ${ }^{31}$

Günümüzde vital veya devital renklenmiş dişlere beyazlatma tedavisi uygulanması sıklıkla kullanılan bir yöntem haline gelmiştir. Beyazlatma tedavilerinin en önemli avantajı doğal diş yapısının bozulmamasıdır. Metal destekli veya tam seramik kron, kompozit veya porselen lamineler gibi geleneksel invaziv yöntemlere başvurulmadan önce, doğru endikasyon doğrultusunda beyazlatma tedavileri uygulanabilir. Ancak hastalar olası servikal kök rezorpsiyonları ve dişin yeniden renklenmesi gibi komplikasyonlar hakkında bilgilendirilmeli ve düzenli aralıklarla kontrol altında tutulmalıdır.

\section{KAYNAKLAR}

1. Hattab FN, Qudeimat MA, AL-RIMAWI HS. Dental discoloration: an overview. J Esthet Dent 1999;11:291-310.

2. Koruk DC, Kırgızoğlu Z. Çocuklarda ve gençlerde diş beyazlatma işlemlerine yaklaşım-derleme. Atatürk Üniv Diş Hek Fak Derg 2010;3:44-53.

3. Watts A, Addy M. Tooth discolouration and staining: Tooth discolouration and staining: a review of the literature. Br Dent J 2001;190:30916.

4. Plotino G, Buono L, Grande NM, Pameijer $\mathrm{CH}$, Somma F. Nonvital tooth bleaching: a review of the literature and clinical procedures. J Endod 2008;34:394-407.

5. Caughman WF, Frazier KB, Haywood VB. Carbamide peroxide whitening of nonvital single discolored teeth: case reports. Quintessence Int 1999;30: 1

6. Feinman RA, Goldstein RE, Garber DA. Bleaching teeth: Quintessence Publishing Company; 1987.

7. MacIsaac AM, Hoen C. Intracoronal bleaching: concerns and considerations. J Can Dent Assoc 1994;60:57-64.

8. Harrington GW, Natkin E. External resorption associated with bleaching of pulpless teeth. J Endod 1979;5:344-8.

9. Friedman S, Rotstein I, Libfeld $H$, Stabholz A, Heling I. Incidence of external root resorption and esthetic results in 58 bleached pulpless teeth. Dent Traumatol 1988;4:23-6.

10. Haywood VB. History, safety, and effectiveness of current bleaching techniques and applications of the nightguard vital bleaching technique. 
Quintessence Int 1992;23: 1

11. Spasser HF. A simple bleaching technique using sodium perborate. NY State Dent J 1961;27:332-4.

12. Weiger R, Kuhn A, Löst C. Effect of various types of sodium perborate on the $\mathrm{pH}$ of bleaching agents. J Endod 1993;19:239-41.

13. Weiger $R$, Kuhn $A$, Löst $C$. In vitro comparison of various types of sodium perborate used for intracoronal bleaching of discolored teeth. J Endod 1994;20:338-41.

14. Hara AT, Pimenta LAF. Nonvital tooth bleaching: A 2-year case report. Quintessence Int 1999;30:1

15. Perrine G, Reichl R, Baisden M, Hondrum S. Comparison of $10 \%$ carbamide peroxide and sodium perborate for intracoronal bleaching. General Dent 1999;48:264-70.

16. Haywood VB, Leech T, Heymann HO, Crumpler D, Bruggers K. Nightguard vital bleaching: effects on enamel surface texture and diffusion. Quintessence Int 1990;21:1

17. Gimlin DR, Schindler WG. The management of postbleaching cervical resorption. J Endod 1990;16:292-7.

18. Madison S, Walton R. Cervical root resorption following bleaching of endodontically treated teeth. J Endod 1990;16:570-4.

19. Lee $G$, Lee $M$, Lum $S$, Poh R, Lim KC. Extraradicular diffusion of hydrogen peroxide and $\mathrm{pH}$ changes associated with intracoronal bleaching of discoloured teeth using different bleaching agents. Int Endod J 2004;37:500-6.

20. Lim M, Lum S, Poh R, Lee G, Lim KC. An in vitro comparison of the bleaching efficacy of $35 \%$ carbamide peroxide with established intracoronal bleaching agents. Int Endod J 2004;37:483-8.

21. Costas FL, Wong M. Intracoronal isolating barriers: effect of location on root leakage and effectiveness of bleaching agents. J Endod 1991;17:365-8.

22. Smith JJ, Cunningham CJ, Montgomery S. Cervical canal leakage after internal bleaching procedures. J Endod 1992;18:476-81.

23. Hansen-Bayless J, Davis R. Sealing ability of two intermediate restorative materials in bleached teeth. Am J Dent 1992;5:151-4.

24. Rotstein I, Zyskind D, Lewinstein I, Bamberger N. Effect of different protective base materials on hydrogen peroxide leakage during intracoronal bleaching in vitro. J Endod 1992;18:114-7.
25. Baratieri LN, Ritter AV, Monteiro Jr S, Caldeira de Andrada MA, Cardoso Vieira LC. Nonvital tooth bleaching: Guidelines for the clinician. Quintessence Int 1995;26:1.

26. Abou-Rass M. Long-term prognosis of intentional endodontics and internal bleaching of tetracyclinestained teeth. Compend Contin Educ Dent 1998;19:1.

27. Dishman MV, Covey DA, Baughan LW. The effects of peroxide bleaching on composite to enamel bond strength. Dent Mater 1994;10:33-6.

28. Torneck C, Titley K, Smith D, Adibfar A. The influence of time of hydrogen peroxide exposure on the adhesion of composite resin to bleached bovine enamel. J Endod 1990;16:123-8.

29. Ruse N, Smith D, Torneck C, Titley K. Preliminary surface analysis of etched, bleached, and normal bovine enamel. J Dent Res 1990;69:1610-3.

30. Kehoe JC. pH reversal following in vitro bleaching of pulpless teeth. J Endod 1987;13:6-9.

31. Demarco F, Freitas J, Silva M, Justino L. Microleakage in endodontically treated teeth: influence of calcium hydroxide dressing following bleaching. Int Endod J 2001;34:495-500

\section{Yazışma Adresi}

Yrd. Doç. Dr. İsmail UZUN

Ondokuz Mayıs Üniversitesi

Diş Hekimliği Fakültesi

Endodonti Anabilim Dalı

SAMSUN

Tel: 05332342355

Email: ismail.uzun@omu.edu.tr 